\title{
Fatores influenciadores do desempenho acadêmico na graduação em administração pública
}

\author{
Jaqueline Zermiani Brandt ${ }^{1}$ \\ ORCID: 0000-0003-2564-5932 \\ Francisca Tejedo-Romero² \\ ORCID: 0000-0002-2600-9826 \\ Joaquim Filipe Ferraz Esteves Araujo ${ }^{3}$ \\ ORCID: 0000-0001-8531-6036
}

\section{Resumo}

A temática do desempenho acadêmico tem despertado interesse de pesquisadores, sobretudo no Brasil, onde as pesquisas são escassas acerca deste tema. De acordo com a literatura, múltiplos fatores podem estar relacionados ao desempenho e influenciá-lo, como por exemplo, os fatores socioeconômicos, de identificação, acadêmicos e ligados às IES e cursos no Brasil. Este trabalho tem como objetivo analisar os fatores que influenciam o desempenho acadêmico dos alunos dos cursos de graduação em administração pública no Brasil, conforme os resultados do Exame Nacional de Desempenho dos Estudantes (Enade) realizado em 2015, ano em que os estudantes dos cursos de administração pública foram avaliados pela primeira vez. A pesquisa de abordagem quantitativa baseia-se no uso de técnicas estatísticas de correlação bivariada e análise univariada. Constatou-se que quanto maior a renda familiar e a escolaridade dos pais melhor foi o desempenho dos estudantes dos cursos de administração pública no Enade. Outros fatores correlacionados positivamente foram: gênero masculino, etnia branca, idade abaixo da média de 32 anos e existência de financiamento (bolsa de estudos). Notou-se ainda que estudar semanalmente mais que três horas e trabalhar vinte ou mais horas semanais contribuiu para um bom desempenho. Os cursos de administração pública estarem localizados na região Sudeste e pertencerem às instituições de ensino superior privadas influenciaram positivamente no desempenho do Enade.

\section{Palavras-chave}

Desempenho acadêmico - Enade - Ensino superior - Administração pública.

1- Universidade do Minho, Braga, Portugal. Contato: jaque.zbrandt@gmail.com.

2- Universidade de Castilla-La Mancha, Albacete, Espanha. Contato: francisca.tejedo@uclm.es.

3- Universidade do Minho, Braga, Portugal e Centro de Investigação em Ciência Política-CICP (UID/CPO/0758/2019). Contato: jilipe@eeg.uminho.pt.

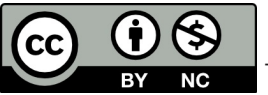

DOl: http://dx.doi.org/10.1590/S1678-4634202046202500

This content is licensed under a Creative Commons attribution-type BY-NC. 


\section{Factors affecting academic achievement by undergraduates in public administration}

\section{Abstract}

The theme of academic achievement has attracted the interest of researchers, especially in Brazil, where studies are scarce on this topic. According to the literature, multiple factors may be related to performance and hold an influence on it, such as socioeconomic, identification, academic, and other factors related to individual higher institutions and their courses in Brazil. This study aims to analyze the factors that influence the academic achievement of undergraduate students in public administration in Brazil, through the National Student Performance Exam (Enade) held in 2015, the year in which the students of the public administration courses were evaluated by the first time. The quantitative approach is based on the use of statistical techniques of bivariate correlation and univariate analysis. It was reported that the higher the family income and the parents' schooling, the better the performance by public administration students in Enade. Other factors refer to male students who obtained the best results, as they are white, under 32 years old and who were funded (with a scholarship). It was also noted that the students' who dedicated more than three weekly hours to study contributed to a good performance, added to twenty or more working hours a week. Public administration courses in the Southeast region delivered by private higher education institutions had a positive influence on Enade's performance.

\section{Keywords}

Academic achievement - Enade - Higher education - Public administration.

\section{Introdução}

0 desempenho acadêmico tem sido um dos indicadores mais estudados quando se trata do (in)sucesso, sendo que diversos pesquisadores indicam que múltiplos fatores podem influenciar o desempenho. Dentre esses fatores estão os socioeconômicos, fatores de identificação, fatores acadêmicos e fatores ligados às instituições de ensino superior (IES) e cursos no Brasil (ALMEIDA et al., 2006; BERTOLIN; MARCON, 2015; TEJEDOR, 2003; TEJEDOR; MUÑOZ-REPISO, 2007). 0 desempenho pode se referir, por exemplo, à classificação de classe, às taxas de sucesso ou conclusão no prazo previsto no currículo e pontuações ou notas obtidas em diferentes disciplinas (MORIANA et al., 2006; SULEYMANOV, 2016; TEJEDOR, 2003; WHITE, 1982). Um dos pontos de debate sobre o desempenho acadêmico está na importância de compreender e identificar os fatores influenciadores, de modo que as IES possam buscar medidas para reduzir o insucesso de seus estudantes. Outro debate está na identificação do que acarreta um bom desempenho, de forma a possibilitar implementar políticas públicas que visem a concretizar a igualdade 
de oportunidades, independentemente da classe social, gênero e etnia dos estudantes (BRITES-FERREIRA et al., 2011; DURÁN SANTOMIL et al., 2016; MÜLLER; PRINSL00; DU PLESSIS, 2007).

No Brasil, são escassas as pesquisas que estudam o desempenho no âmbito do ensino superior, sobretudo dos cursos de administração pública, cuja primeira avaliação do desempenho dos alunos do curso ocorreu em 2015 no Exame Nacional de Desempenho dos Estudantes (Enade). Desde 2004, utiliza-se o Enade para avaliar o desempenho dos alunos em relação aos conteúdos programáticos da respectiva área de graduação, suas habilidades e competências aos temas externos específicos da sua profissão (BRASIL, 2009).

Dessa forma, o artigo busca analisar os fatores que influenciam o desempenho acadêmico dos alunos dos cursos de graduação em administração pública no Brasil, de modo a responder à questão de partida: QP1. Quais fatores influenciam o desempenho acadêmico dos alunos de graduação em administração pública no Brasil?

A pesquisa de abordagem quantitativa se baseia no uso de técnicas estatísticas de correlação bivariada e análise univariada. Os resultados apontam para a correlação do desempenho acadêmico com os fatores: renda, escolaridade dos pais, etnia, gênero, idade, horas de estudo, financiamento, horas de trabalho, categoria administrativa e região de funcionamento do curso.

\section{Desenvolvimento teórico e desenvolvimento de hipóteses}

\section{Fatores socioeconômicos}

0 primeiro estudo que buscou entender os determinantes do desempenho escolar, foi o Relatório Equality of educational opportunity. 0 achado principal foi de que o background dos alunos, ou seja, as características socioeconômicas eram os principais determinantes das diferenças em seus desempenhos (COLEMAN et al., 1966). Desde lá, são inúmeras as pesquisas que buscam verificar a relação entre o background dos alunos e seu desempenho na educação básica. Já no ensino superior essas pesquisas são escassas (BERTOLIN; MARCON, 2015).

Apesar de a literatura indicar os fatores ou determinantes que influenciam o desempenho acadêmico, esses podem ser variados (ALMEIDA et al., 2005; STEENKAMP; BAARD; FRICK, 2009; TEJEDOR, 2003), desde as características socioeconômicas e culturais dos indivíduos e de suas famílias, até as dinâmicas que ocorrem nas salas de aula, entre professores e alunos, e as características estruturais da escola (PALERMO; SILVA; NOVELLINO, 2014). Dessa forma, "assume que os estudantes trazem consigo uma variedade de atributos (gênero e capacidade), origem familiar (status social, expectativas familiares) e experiências pré-universitárias (sucesso no ensino médio e/ou outros estudos prévios)" (FAGUNDES, 2014, p. 513).

No Brasil, as pesquisas focadas nos fatores influenciadores do desempenho acadêmico dialogam com os fatores encontrados na realidade de outros países como gênero, status socioeconômico e fatores pedagógicos/acadêmicos (ALBERNAZ; FERREIRA; FRANCO, 2002; BARBOSA; FERNANDES, 2001; CÉSAR; SOARES, 2001). 
Dentre os diversos fatores que estão ligados ao desempenho acadêmico, os socioeconômicos têm sido os mais estudados, sobretudo quando se trata de renda familiar e escolaridade e/ou profissão dos pais. Há também outras variáveis utilizadas e que compõem o nível socioeconômico, como, por exemplo, a etnia (SIRIN, 2005; WHITE, 1982).

Para os fatores sócio familiares ou socioeconômicos, a teoria do handicap sociocultural (BERNSTEIN, 2003) explica que o desempenho acadêmico não depende apenas de causas psicológicas individuais (teoria dos dotes), mas de diferenças entre estratos sociais da família (BENAVENTE, 1990; FERNANDES, 1991; TOMÉ, 2013; LEMOS, 2013; PINTO; DELGAD0; MARTINS, 2015). 0 estrato social familiar proporcionaria ou não motivações e estímulos para a aprendizagem e melhor desempenho (BENAVENTE, 1990).

Das diversas pesquisas que trazem os fatores socioeconômicos como determinantes do desempenho acadêmico, um recorte delas se inicia pela pesquisadora Saavedra (2001), que buscou analisar se havia relação entre as classificações escolares e o nível socioeconômico de alunos. Os resultados apontaram que, para os alunos com altas classificações em todas as disciplinas, os pais possuíam alto grau de escolaridade e exerciam profissões de formação acadêmica. Notou-se também que as piores classificações advinham de alunos cujos pais possuíam baixa escolaridade (SAAVEDRA, 2001). Em outra pesquisa, encontrou-se que o nível de escolaridade dos pais está associado a um melhor desempenho dos alunos em matemática, leitura e ciência. Porém, essa associação é indireta, a escolaridade dos pais é um forte determinante dos seus status ocupacionais (profissão), o que lhes permite oferecer aos filhos maior acesso aos recursos educacionais e culturais (POKROPEK; BORGONOVI; JAKUBOWSKI, 2015). Em relação à renda familiar, os graduandos concluintes pertencentes a famílias com renda abaixo de três salários mínimos, apresentam, em geral, um desempenho inferior ao dos estudantes com renda familiar maior que três salários mínimos. Conforme a renda mensal familiar aumenta, a média geral dos estudantes concluintes aumenta (BERTOLIN; MARCON, 2015). Em estudo longitudinal, também se concluiu que as condições socioeconômicas das famílias estão ligadas ao desempenho acadêmico dos alunos. Os resultados apontaram que alunos de famílias de baixo nível socioeconômico (NSE) não estão apenas expostas a um ambiente familiar desfavorável, por terem baixo monitoramento dos pais em atividades escolares, mas também podem experimentar baixo sucesso acadêmico na escola (BAE; WICKRAMA, 2015).

Da mesma forma, a variável etnia pode estar relacionada com o desempenho acadêmico. Os achados em estudo que buscou identificar a existência de relação entre desempenho e etnia, demonstrou que estudantes brancos atuaram melhor do que afro-americanos e latinos. Além disso, os asiáticos e os latinos possuíam um autoconceito de matemática mais elevado do que os afro-americanos (DEFREITAS; RINN, 2013). Outro estudo também indicou que estudantes euro-americanos possuem melhor desempenho do que estudantes afro-americanos. Entretanto, houve diminuição dessa diferença no desempenho entre etnias ao se fortalecer o senso de pertença social dos estudantes afro-americanos. 0 estudo ressaltou que, para explicar essas diferenças de desempenho, deve-se levar em consideração as origens das desigualdades que determinados grupos étnicos enfrentam na sociedade. Essas desigualdades podem ser atribuídas a fatores psicológicos ligados ao senso de pertença social. Esse senso de pertença social consiste em um indivíduo possuir uma inter-relação positiva com outros indivíduos (WALTON; COHEN, 2011). 
Os pesquisadores Silva e colaboradores (2010) notaram que o desempenho foi ligeiramente maior a favor dos brancos na formação geral, nos cursos biologia, física, geografia, história, letras, matemática, pedagogia e química. No componente específico, verificou-se igualmente melhor desempenho entre os brancos, nos cursos de arquitetura e urbanismo, ciências sociais, filosofia, física, geografia, história, letras, matemática, pedagogia e química (SILVA; VENDRAMINI; LOPES, 2010). Nesse contexto, este estudo pretende investigar as seguintes hipóteses:

H1: Há uma relação entre a renda familiar e o desempenho acadêmico dos estudantes de administração pública?

H2: Há uma relação entre a escolaridade dos pais e o desempenho acadêmico dos estudantes de administração pública?

H3: Há uma relação entre a etnia dos estudantes de administração pública e seu desempenho acadêmico?

Além dos fatores socioeconômicos (renda, escolaridade dos pais e etnia) mais relacionados como influenciadores no desempenho acadêmico, há também os fatores de identificação como gênero e idade que interferem no desempenho dos estudantes e são contextualizados a seguir.

\section{Fatores de identificação (gênero e idade)}

Conforme mencionado, apesar de os fatores determinantes do desempenho acadêmico serem múltiplos, várias pesquisas identificam e categorizam as características pessoais, ou seja, gênero e idade, como variáveis que estão relacionadas ao desempenho. Apesar de as conclusões serem diversas em relação ao gênero, nota-se que, dependendo da área e outros fatores ligados aos estudantes, como escolaridade dos pais ou abandono escolar, há disparidade entre os desempenhos de mulheres e homens. Em relação à idade, pesquisas apontam melhor rendimento entre alunos mais jovens (MÜLLER; PRINSLO0; DU PLESSIS, 2007; TEJEDOR, 2003).

As estudantes do gênero feminino, mesmo em desvantagem socioeconômica, se igualam ou, às vezes, superam os alunos do gênero masculino, mesmo em disciplinas comumente consideradas de melhor desempenho masculino, como por exemplo físicoquímica e matemática. Por outro lado, têm desempenho inferior na disciplina de educação física (SAAVEDRA, 2001). As estudantes que possuem pelo menos um dos pais com formação superior apresentaram notas mais elevadas na universidade, ao final do primeiro ano de licenciatura, do que os estudantes do gênero masculino, com pais apenas com a educação básica (ALMEIDA et al., 2006). Em pesquisa de Buchmann e DiPrete (2006) os achados indicaram a vantagem das alunas na conclusão do ensino superior. 0 fator primário dessa vantagem é ocasionado por uma maior taxa de abandono da graduação pelos estudantes do gênero masculino, cujas famílias possuem pai ausente ou com nível baixo de escolaridade (BUCHMANN; DIPRETE, 2006). Outros estudos também demonstraram que estudantes do gênero feminino apresentaram melhores resultados na realidade universitária do que estudantes do gênero masculino (MILLS et al., 2009; PÉREZ-CÁRCELES et al., 2014). Em pesquisa, Silva, Vendramini e Lopes, (2010) verificaram 
que o desempenho dos estudantes no Enade variou segundo o gênero. Primeiramente, estudantes do gênero masculino obtiveram desempenho médio significativamente superior ao de estudantes do gênero feminino na parte de formação geral, nos cursos de matemática, letras, biologia, história, geografia e filosofia. Nessa mesma parte da prova, estudantes do gênero feminino obtiveram médias estatisticamente maiores que estudantes do gênero masculino nos cursos de arquitetura e urbanismo, ciências sociais e engenharias. No componente específico do exame, estudantes do gênero masculino obtiveram médias estatisticamente maiores que os do gênero feminino nos cursos de matemática, letras, física, biologia, história, geografia, filosofia, computação e engenharias. Nessa mesma parte do exame, estudantes do gênero feminino obtiveram médias estatisticamente maiores que estudantes do gênero masculino nos cursos de pedagogia e ciências sociais. Esses resultados trazem a reflexão sobre a inversão de papéis em áreas marcadas por estereótipos. Por exemplo, em cursos como letras, biologia, filosofia entre outros, em que houve uma diferença a favor dos homens. Outro destaque está na parte do exame de formação geral, em que as médias das mulheres em áreas consideradas masculinas, como engenharia e arquitetura, foram maiores (SILVA; VENDRAMINI; LOPES, 2010).

Em relação à idade, estudantes mais jovens têm mais sucesso do que estudantes mais velhos (MÜLLER; PRINSLO0; DU PLESSIS, 2007). Em concordância, o desempenho dos alunos que cursaram o ensino superior na idade de até 24 anos foi melhor (MORICONI; NASCIMENTO, 2014). Em estudo similar, o fator idade influenciou negativamente a nota bruta do Enade de 2012 dos alunos de ciências contábeis, ou seja, quanto mais velhos os alunos, menores foram as suas notas (CARMO; ALMEIDA, 2015). Nesse contexto, este estudo pretende investigar as seguintes hipóteses:

H4: Há uma relação entre o gênero dos estudantes de administração pública e seu desempenho acadêmico?

H5: Há uma relação entre a idade dos estudantes de administração pública e seu desempenho acadêmico?

Conforme descrito anteriormente, alguns dos fatores que podem influenciar o desempenho acadêmico são os socioeconômicos e de identificação. Entretanto, outros fatores como quantidade de horas dedicadas aos estudos, financiamento e trabalho também podem estar intimamente ligados ao desempenho dos estudantes. 0 tópico a seguir trata sobre o terceiro fator apontado na literatura, o acadêmico.

\section{Fatores acadêmicos}

Em relação aos fatores acadêmicos, apesar de os resultados serem diversos, algumas variáveis são descritas como influenciadoras do desempenho dos estudantes, como quantidade de horas dedicadas aos estudos, existência de ajuda financeira ou financiamento (bolsa de estudos), tempo dedicado ao trabalho, dentre outros (DURÁN SANTOMIL et al., 2016).

Em pesquisas que buscaram analisar a variável horas de estudo, os resultados apontaram para essa variável como influenciadora do desempenho acadêmico (ANDRIETTI; VELASC0, 2015). Os pesquisadores Durán Santomil e colaboradores (2016) notaram que 
estudantes que estudaram entre trinta e quarenta horas obtiveram, em média, uma nota final superior aos que estudavam menos de dez horas (DURÁN SANTOMIL et al., 2016).

Quanto ao financiamento, não há consenso quanto a existência de relação com o desempenho. Stater (2009), em pesquisa que buscou examinar os efeitos do financiamento no primeiro e último ano da faculdade sobre desempenho acadêmico, encontrou resultados que indicam uma relação positiva. Já outro estudo indicou relação negativa entre a quantidade de ajuda recebida e o desempenho acadêmico dos estudantes, sugerindo que esta ajuda não é suficiente para compensar as necessidades de estudantes com maior dificuldade financeira (CORIA; HOFFMAN, 2016). Embora estudos anteriores tenham encontrado relação entre desempenho acadêmico e financiamento, outros autores não encontraram relações estatisticamente significativas (COONROD, 2008; SCOTTCLAYTON, 2011).

Em relação à influência das horas de trabalho remunerado no desempenho acadêmico, estudos indicam que quanto mais horas trabalhadas, maiores eram as chances de um efeito negativo no desempenho do estudante. Ou seja, um estudante que trabalha tem menos chances de conseguir um bom desempenho do que estudantes que não trabalham (CALLENDER, 2008; CARNEY; McNEISH; McCOLL, 2005). Entretanto, alguns pesquisadores indicam não haver uma relação estatística significativa entre o trabalho e as notas dos alunos (DAROLIA, 2014; DURÁN SANTOMIL et al., 2016). Nesse contexto, este estudo pretende investigar as seguintes hipóteses:

H6: Há uma relação entre as horas de estudo semanais dedicadas pelos estudantes de administração pública e seu desempenho acadêmico?

H7: Há uma relação entre o financiamento recebido pelos estudantes de administração pública e seu desempenho acadêmico?

H8: Há uma relação entre as horas de trabalho dos estudantes de administração pública e seu desempenho acadêmico?

Os fatores acadêmicos, de identificação e socioeconômicos são apontados por pesquisas no Brasil e no mundo como influenciadores do desempenho acadêmico. No Brasil, outros fatores foram identificados, como os ligados às instituições de ensino superior e dos próprios cursos de administração pública, objeto deste estudo. Encerra-se assim, no tópico a seguir a discussão dos fatores elencados sobre o debate do desempenho acadêmico.

\section{Fatores ligados às características das IES e cursos de administração pública}

Os últimos fatores elencados como influenciadores do desempenho acadêmico encontrados em pesquisas brasileiras referem-se às categorias administrativas (privada ou pública) das instituições de ensino superior e também à região em que o curso se localiza.

Ao buscar entender a variável categoria administrativa, notou-se que os estudantes de IES públicas apresentaram melhores resultados no Enade do que os estudantes de IES privadas (CARMO; ALMEIDA, 2015; LEMOS; MIRANDA, 2015). Em outra investigação, os pesquisadores Crepalde e Silveira (2016) concluíram que os estudantes de IES privadas tiveram melhor desempenho do que os alunos de IES públicas (CREPALDE; SILVEIRA, 2016). 
A região em que o curso se localiza também é uma variável que pode influenciar o desempenho. De acordo com os achados de Carmo e Almeida (2015), o fato de o curso se situar na região Sul pode ter influenciado positivamente o desempenho dos estudantes de ciências contábeis no Enade. Por outro lado, a nota da prova de formação geral pode ter caído pelo fato de o estudante pertencer a cursos de ciências contábeis localizados na região Norte (CARMO; ALMEIDA, 2015). As hipóteses a serem investigadas são:

- Há uma relação entre a categoria administrativa das IES e o desempenho acadêmico dos estudantes de administração pública?

- Há uma relação entre a região do curso e o desempenho acadêmico dos estudantes de administração pública?

\section{Metodologia da pesquisa}

Esta seção apresenta a amostra utilizada e justifica as variáveis selecionadas para testar a hipótese do estudo empírico.

\section{Amostra}

0 universo considerado nesta pesquisa são todos os alunos concluintes do curso de administração pública, regularmente inscritos no Enade 2015 e que compareceram à prova, resultando numa amostra de 3.303 estudantes. Entretanto, desses, foram desconsiderados os estudantes que não apresentaram informações para todas as variáveis analisadas e os casos extremos, resultando numa amostra de 3.263 alunos. Esses estudantes estavam matriculados em IES públicas e privadas, em todas as regiões do país (Norte e Nordeste, Centro-Oeste, Sudeste e Sul).

\section{Variáveis e coleta de dados}

A fonte de informação dos dados desta pesquisa é secundária, com base nos microdados estatísticos externos coletados e disponibilizados pelo Instituto Nacional de Estudos e Pesquisas Educacionais Anísio Teixeira (Inep) em 2015 ao aplicar o Enade aos estudantes de graduação no Brasil.

Variável dependente. A nota bruta da prova é a média ponderada da formação geral (25\%) e do componente específico (75\%). É uma variável contínua que assume os valores entre 0 e 100. Nesta investigação, a nota bruta da prova é vista como uma proxy ao desempenho acadêmico dos estudantes (concluintes) de administração pública no Enade 2015.

Variáveis independentes. Foram selecionadas as variáveis que podem influenciar o desempenho acadêmico, variáveis de acordo com as características das IES/cursos e dos estudantes. Tendo em vista a metodologia quantitativa adotada nesta pesquisa, todas as variáveis relacionadas às características das IES/cursos e dos estudantes foram convertidas em variáveis dummies, com exceção da variável idade. 
Renda familiar. Variável categórica que representa a classe social de renda mensal das pessoas que moram na casa com o estudante, de acordo com: 1 = classe baixa; $2=$ classe média; e 3 = classes média alta e alta. Foram considerados dummies para cada classe de renda, assumindo valor 1 se a renda familiar pertence a uma determinada classe em questão e valor 0 em caso contrário. A divisão das classes sociais é definida segundo os seguintes critérios: classe alta e média alta = acima de dez salários mínimos (mais de R\$ 7.240,01); classe média = de 4,5 a dez salários mínimos ( $\mathrm{R} \$ 3.258,01$ a $\mathrm{R} \$$ 7.240,00); e classe baixa = Até de 4,5 salários mínimos (até R\$ 3.258,00).

Escolaridade dos pais. Variável categórica que representa o nível de escolaridade dos pais de acordo com: 1 = nenhuma escolaridade; 2 = ensino fundamental (inclui do $1^{\circ}$ ao $9^{\circ}$ ano); 3 = ensino médio; 4 = ensino superior (inclui pós-graduação). Foram considerados dummies para cada nível de escolaridade, assumindo valor 1 se o nível de escolaridade pertence a um determinado nível e valor 0 em caso contrário.

Etnia. Variável categórica que representa a etnia do estudante de acordo com: 1 = branco(a); 2 = negro(a) e 3 = outros(as). Foram considerados dummies para cada etnia, assumindo valor e 1 se o estudante pertence a uma etnia determinada e valor 0 em caso contrário.

Gênero. Variável binária que representa o gênero dos estudantes. É uma variável dummy que assume valor 1 para caso o estudante seja do gênero masculino e 0 caso seja do gênero feminino.

Idade. Variável contínua que representa a idade do estudante no dia do Enade. Essa variável é medida como o logaritmo natural do número de anos.

Horas de estudo. Variável categórica que representa o número de horas por semana dedicadas aos estudos, de acordo com: 1 = nenhuma; 2 = uma a três horas; e 3 = mais de três horas. Foram considerados dummies para cada dimensão, assumindo valor 1 para as horas de estudo pertencente a uma determinada dimensão e valor 0 em caso contrário.

Financiamento. Variável binária que representa se o estudante possui algum tipo de financiamento em seus estudos. É uma variável dummy que assume valor 1 para o caso de possuir financiamento e 0 em caso contrário.

Horas de trabalho. Variável categórica que representa a carga horária de trabalho remunerado do estudante, de acordo com: 1 = não trabalha; 2 = trabalha eventualmente e 3 = trabalha vinte ou mais horas. Foram considerados dummies para uma das dimensões, assumindo valor 1 se o estudante pertence a uma determinada dimensão e valor 0 em caso contrário.

Categoria administrativa. Variável binária que representa a categoria administrativa da IES. É uma variável dummy que assume valor 1 para o caso de IES privada e 0 em caso de IES pública.

Região do curso. Variável categórica que representa a região de funcionamento do curso de acordo com: 1 = Norte e Nordeste; 2 = Sudeste; 3 = Sul e 4 = Centro-Oeste. Foram considerados dummies para cada uma das regiões, assumindo valor 1 se o funcionamento do curso pertence a uma região determinada e valor 0 em caso contrário. 


\section{Resultados e discussão}

Esta seção apresenta os resultados empíricos do estudo por meio de duas análises. Primeiramente, estudou-se a correlação bivariada entre a variável dependente e as independentes. Posteriormente, realizou-se uma análise univariada mediante contraste individual de cada uma das hipóteses da pesquisa, com a intenção de determinar a possível existência de diferenças significativas entre os grupos.

\section{Análise bivariada}

A seguir, serão analisadas as possíveis relações entre a variável dependente e as variáveis independentes. Além disso, avalia-se a possível existência de multicolinearidade entre todas as variáveis. Na Tabela 1, são apresentados os coeficientes da matriz de correlação de Spearman.

Tabela 1 - Matriz de correlações de Spearman

\begin{tabular}{|c|c|c|c|c|c|c|c|c|c|c|c|}
\hline & (1) & (2) & (3) & (4) & (5) & (6) & (7) & (8) & (9) & (10) & (11) \\
\hline $\begin{array}{c}\text { (1) Nota bruta da } \\
\text { prova }\end{array}$ & 1 & & & & & & & & & & \\
\hline (2) Renda & $0,319^{\star *}$ & 1 & & & & & & & & & \\
\hline $\begin{array}{c}\text { (3) } \\
\text { Escolaridade dos pais }\end{array}$ & $0,172^{\star \star}$ & $0,264^{* *}$ & 1 & & & & & & & & \\
\hline (4) Etnia & $-0,142^{* *}$ & $-0,194^{\text {** }}$ & $-0,163^{\star *}$ & 1 & & & & & & & \\
\hline (5) Gênero & $0,187^{* *}$ & $0,211^{\star *}$ & $0,036^{\star}$ & $-0,021$ & 1 & & & & & & \\
\hline (6) Idade & $-0,083^{\star \star}$ & 0,003 & $-0,318^{\star \star}$ & $0,096^{* *}$ & $0,115^{\star \star}$ & 1 & & & & & \\
\hline (7) Horas de estudo & $0,129^{* *}$ & $0,037^{*}$ & $-0,016$ & 0,002 & 0,010 & $0,060^{\star *}$ & 1 & & & & \\
\hline (8) Financiamento & 0,032 & $-0,011$ & $0,073^{* *}$ & $-0,018$ & 0,018 & $-0,158^{* *}$ & $0,049^{* *}$ & 1 & & & \\
\hline (9) Horas de trabalho & $-0,049^{* *}$ & 0,023 & $-0,195^{\star \star}$ & $0,058^{\star \star}$ & $0,104^{\star *}$ & $0,415^{\star *}$ & $-0,012$ & $-0,140^{\star *}$ & 1 & & \\
\hline $\begin{array}{c}\text { (10) Categoria } \\
\text { administrativa da IES }\end{array}$ & $0,072^{* *}$ & $0,084^{\star *}$ & $0,122^{\star *}$ & $-0,048^{\star *}$ & 0,002 & $-0,151^{* *}$ & 0,026 & $0,302^{\star *}$ & $-0,110^{* *}$ & 1 & \\
\hline $\begin{array}{l}\text { (11) Região de } \\
\text { funcionamento do } \\
\text { curso }\end{array}$ & $-0,092^{\star *}$ & $0,131^{\star *}$ & $-0,001$ & $-0,193^{\star *}$ & $-0,073^{\star *}$ & 0,008 & $-0,004$ & $-0,027$ & $0,069^{* *}$ & $-0,025$ & 1 \\
\hline
\end{tabular}

Fonte: elaboração própria com base nos dados da pesquisa.

** A correlação é significativa no nível 0,01 (bilateral).

${ }^{*}$ A correlação é significativa no nível 0,05 (bilateral). 
A nota bruta da prova está correlacionada positivamente a um nível de $1 \%$ com a renda, escolaridade dos pais, gênero, horas de estudo e categoria administrativa. Por outro lado, a nota bruta da prova está correlacionada negativamente a um nível de $1 \%$ com etnia, idade, horas de trabalho e região de funcionamento do curso. No entanto, não foi possível confirmar, a um nível de significação de 1 e 5\%, uma correlação entre a nota bruta da prova e a variável financiamento.

Além disso, os valores não indicaram colinearidade. Um problema de colinearidade é considerado severo se um coeficiente de correlação entre pares for maior que 0,80 (GUJARATI, 1995).

\section{Análise univariada}

0 objetivo desta análise é determinar aqueles fatores que influenciam o desempenho acadêmico. Dessa forma, aplica-se um contraste de diferenças médias para a variável dependente com a variável independente. Assim, para realizar esses contrastes, foram formados dois grupos de amostras com a finalidade de comparar os valores médios do desempenho. Esses grupos são criados por classificação da amostra a partir das variáveis independentes estudadas. Neste caso, a variável independente é a variável de agrupação na função de um determinado critério (as categorias da variável). Para a variável idade, que é contínua, o agrupamento se deu em função do valor da média da referida variável $(32,696)$. Assim, formaram-se dois grupos: um composto por sujeitos de idade igual ou superior à média e outro por aqueles de idade abaixo da média. Em primeiro lugar, para determinar a aplicação de um contraste paramétrico ou não paramétrico, fez-se necessário verificar os pressupostos de normalidade e homocedasticidade das varáveis independentes mediante a prova de Kolmogorov-Smirnov e o teste de Levene. Como se pode observar na Tabela 2, a maioria das variáveis não cumprem esses pressupostos. Sendo assim, realizaram-se testes não paramétricos.

Desse modo, como apresentado na Tabela 3, para as amostras independentes com um máximo de duas categorias, procedeu-se a análise da diferença de médias aplicando o Teste U de Mann-Whitney e o estatístico W da soma de classificação de Wilcoxon, que contrastam se as amostras são equivalentes na sua posição. Para a amostra com mais de duas categorias foi utilizado o Teste de Kruskal-Wallis. 
Tabela 2 - Provas de normalidade e homogeneidade da variância

\begin{tabular}{|c|c|c|c|c|c|c|c|}
\hline & & \multicolumn{4}{|c|}{ Provas de normalidade } & \multirow{2}{*}{\multicolumn{2}{|c|}{$\begin{array}{c}\text { Prova de homogeneidade } \\
\text { da variância }\end{array}$}} \\
\hline & & \multicolumn{2}{|c|}{ Kolmogorov-Smirnov ${ }^{\mathrm{a}}$} & \multicolumn{2}{|c|}{ Shapiro-Wilk } & & \\
\hline & & Estatístico & Sig. & Estatístico & Sig. & Estatístico & Sig. \\
\hline \multirow{4}{*}{ Desempenho } & Renda familiar & & & & & \multirow{4}{*}{0,422} & \multirow{4}{*}{0,656} \\
\hline & Classe baixa & 0,024 & 0,012 & 0,996 & 0,000 & & \\
\hline & Classe média & 0,043 & 0,000 & 0,984 & 0,000 & & \\
\hline & Classe alta e média alta & 0,079 & 0,000 & 0,953 & 0,000 & & \\
\hline \multirow{5}{*}{ Desempenho } & Escolaridade dos pais & & & & & \multirow{5}{*}{1,659} & \multirow{5}{*}{0,174} \\
\hline & Nenhum & 0,048 & $0,200^{*}$ & 0,991 & 0,391 & & \\
\hline & Ensino fundamental & 0,018 & $0,200^{*}$ & 0,997 & 0,009 & & \\
\hline & Ensino médio & 0,036 & 0,005 & 0,986 & 0,000 & & \\
\hline & Ensino superior & 0,043 & 0,005 & 0,984 & 0,000 & & \\
\hline \multirow{4}{*}{ Desempenho } & Etnia & & & & & \multirow{4}{*}{0,359} & \multirow{4}{*}{0,698} \\
\hline & Branco(a) & 0,029 & 0,004 & 0,991 & 0,000 & & \\
\hline & Negro(a) & 0,025 & 0,019 & 0,994 & 0,000 & & \\
\hline & Outros(as) & 0,087 & $0,200^{*}$ & 0,976 & 0,278 & & \\
\hline \multirow{3}{*}{ Desempenho } & Gênero & & & & & \multirow{3}{*}{0,460} & \multirow{3}{*}{0,498} \\
\hline & Feminino & 0,025 & 0,014 & 0,996 & 0,000 & & \\
\hline & Masculino & 0,031 & 0,002 & 0,988 & 0,000 & & \\
\hline \multirow{3}{*}{ Desempenho } & Idade & & & & & \multirow{3}{*}{1,088} & \multirow{3}{*}{0,297} \\
\hline & Inferior à média 32,696 & 0,029 & 0,001 & 0,992 & 0,000 & & \\
\hline & Superior à média & 0,035 & 0,000 & 0,993 & 0,000 & & \\
\hline \multirow{4}{*}{ Desempenho } & Horas de estudo & & & & & \multirow{4}{*}{0,179} & \multirow{4}{*}{0,836} \\
\hline & Nenhuma & 0,077 & 0,126 & 0,984 & 0,214 & & \\
\hline & Uma a três horas & 0,022 & 0,109 & 0,996 & 0,000 & & \\
\hline & Mais de três horas & 0,035 & 0,000 & 0,989 & 0,000 & & \\
\hline \multirow{3}{*}{ Desempenho } & Financiamento & & & & & \multirow{3}{*}{15,056} & \multirow{3}{*}{0,000} \\
\hline & Não tem financiamento & 0,025 & 0,000 & 0,993 & 0,000 & & \\
\hline & Tem financiamento & 0,062 & $0,200^{*}$ & 0,971 & 0,009 & & \\
\hline & Horas de trabalho & & & & & & \\
\hline Decomnenhe & Trabalha vinte horas ou mais & 0,042 & 0,006 & 0,984 & 0,000 & 5006 & 0,007 \\
\hline Deseminpermo & Trabalha eventualmente & 0,052 & $0,200^{*}$ & 0,988 & 0,321 & 0,000 & 0,001 \\
\hline & Não trabalha & 0,023 & 0,005 & 0,995 & 0,000 & & \\
\hline & Categoria Administrativa & & & & & & \\
\hline Desempenho & IES pública & 0,023 & 0,000 & 0,994 & 0,000 & 3,573 & 0,059 \\
\hline & IES privada & 0,168 & 0,003 & 0,871 & 0,000 & & \\
\hline & Região do Curso & & & & & & \\
\hline & Norte e Nordeste & 0,021 & $0,200^{*}$ & 0,997 & 0,020 & & \\
\hline Desempenho & Sudeste & 0,042 & 0,000 & 0,973 & 0,000 & 0,121 & 0,948 \\
\hline & Sul & 0,048 & $0,200^{*}$ & 0,986 & 0,031 & & \\
\hline & Centro-Oeste & 0,027 & $0,200^{*}$ & 0,996 & 0,057 & & \\
\hline
\end{tabular}

Fonte: elaboração própria com base nos dados da pesquisa.

a Correção de significância de Lilliefors.

* Este é um limite inferior da significação verdadeira. 
Tabela 3 - Resultados da análise univariada

\begin{tabular}{|c|c|c|c|c|c|c|c|}
\hline & Variáveis & $N$ & $\begin{array}{l}\text { Intervalo } \\
\text { médio }\end{array}$ & Qui-quadrado ${ }^{\mathrm{a}}$ & $\begin{array}{c}\text { U de } \\
\text { Mann-Whitney }\end{array}$ & $\begin{array}{c}\text { W de } \\
\text { Wilcoxon }\end{array}$ & $Z$ \\
\hline \multirow{4}{*}{ Renda } & Classe baixa & 1.921 & $1.392,15$ & \multirow{4}{*}{$333,708^{* * *}$} & & & \\
\hline & Classe média & 973 & $1.887,22$ & & & & \\
\hline & Classe alta e média alta & 369 & $2.207,68$ & & & & \\
\hline & Nenhum & 168 & $1.305,75$ & & & & \\
\hline \multirow{3}{*}{$\begin{array}{c}\text { Escolaridade } \\
\text { dos pais }\end{array}$} & Fundamental & 1.475 & $1.477,23$ & & & & \\
\hline & Médio & 953 & $1.694,23$ & & & & \\
\hline & Superior & 667 & $1.967,53$ & & & & \\
\hline \multirow{3}{*}{ Etnia } & Branco(a) & 1.591 & $1.768,66$ & \multirow{3}{*}{$65,817^{\star \star \star}$} & & & \\
\hline & Negro(a) & 1.611 & $1.505,06$ & & & & \\
\hline & Outros(as) & 61 & $1.420,03$ & & & & \\
\hline \multirow{2}{*}{ Gênero } & Feminino & 1.765 & $1.469,93$ & & \multirow{2}{*}{1035924,5} & \multirow{2}{*}{2594419,5} & \multirow{2}{*}{$-10,667^{\star \star \star}$} \\
\hline & Masculino & 1.498 & $1.822,96$ & & & & \\
\hline \multirow{2}{*}{ Idade } & Inferior à média 32,696 & 1.830 & $1.667,39$ & & \multirow{2}{*}{1246434,5} & \multirow{2}{*}{2273895,5} & \multirow{2}{*}{$-2,425^{\star \star}$} \\
\hline & Superior à média & 1.433 & $1.586,81$ & & & & \\
\hline \multirow{3}{*}{$\begin{array}{l}\text { Horas de } \\
\text { estudo }\end{array}$} & Nenhuma & 107 & $1.472,18$ & \multirow{3}{*}{$54,751^{\star \star \star}$} & & & \\
\hline & Uma a três & 1.449 & $1.506,71$ & & & & \\
\hline & Mais de três & 1.707 & $1.748,37$ & & & & \\
\hline \multirow{2}{*}{ Financiamento } & Não tem financiamento & 3.136 & $1.625,99$ & & \multirow{2}{*}{180275} & \multirow{2}{*}{5099091} & \multirow{2}{*}{$-1,812^{*}$} \\
\hline & Tem financiamento & 127 & $1.780,51$ & & & & \\
\hline \multirow{3}{*}{$\begin{array}{l}\text { Horas de } \\
\text { trabalho }\end{array}$} & Não estou trabalhando & 2.457 & $1.607,59$ & \multirow{3}{*}{$12,439^{\star \star \star}$} & & & \\
\hline & Trabalho eventualmente & 133 & $1.527,28$ & & & & \\
\hline & $\begin{array}{l}\text { Trabalho vinte horas ou } \\
\text { mais }\end{array}$ & 673 & $1.741,82$ & & & & \\
\hline \multirow{2}{*}{$\begin{array}{c}\text { Categoria } \\
\text { administrativa }\end{array}$} & Pública & 3.218 & $1.624,00$ & & \multirow{2}{*}{46662} & \multirow{2}{*}{5226033} & \multirow{2}{*}{$-4,102^{\star \star *}$} \\
\hline & Privada & 45 & $2.204,07$ & & & & \\
\hline \multirow{4}{*}{ Região } & Norte e Nordeste & 1.153 & $1.506,82$ & \multirow{4}{*}{$497,093^{\text {***}}$} & & & \\
\hline & Sudeste & 1.213 & $2.041,57$ & & & & \\
\hline & Sul & 222 & $1.763,51$ & & & & \\
\hline & Centro-oeste & 675 & $1.066,57$ & & & & \\
\hline
\end{tabular}

Fonte: elaboração própria com base nos dados da pesquisa.

Nota: ${ }^{\star} p<0,1 ;{ }^{* \star} p<0,05 ;{ }^{* \star *} p<0,01$; a. Teste de Kruskal Wallis.

Os resultados apresentados na Tabela 3 revelam que a renda (H1) influencia o desempenho a um nível de significância de 1\%. Existem importantes discrepâncias entre as distintas classes de renda quando se aplica o Teste Kruskal-Wallis, determinando que essas classes não são iguais, e os estudantes da classe alta e média alta obtiveram melhor desempenho. Portanto, existem diferenças significativas entre as classes de renda 
analisadas de acordo com seu nível de desempenho, obtendo um $\chi 2$ igual a 333,708, com um valor $\mathrm{p} \leq 0,01$. Dessa forma, confirma-se a H1.

Para a variável escolaridade dos pais, também ocorreu uma forte diferença entre as dimensões. Ao aplicar o Teste Kruskal-Wallis, nota-se que os estudantes cujos pais tem nível superior obtêm maiores notas. 0 valor calculado para $\chi 2$ foi igual a 148,723, com um valor $\mathrm{p} \leq 0,01$. Assim, aceita-se a $\mathrm{H} 2$.

Os resultados refletem que os estudantes de classe alta e média alta e cujos pais possuem nivel superior obtiveram melhor desempenho. Na literatura, diferentes pesquisas utilizam a renda familiar e escolaridade dos pais para compor a variável denominada de nível socioeconômico (NSE). Nessas pesquisas, os resultados apontaram que os estudantes com alto NSE obtiveram melhor desempenho (BAE; WICKRAMA, 2015; BERTOLIN; MARCON, 2015). Isso indica que renda familiar e escolaridade dos pais exercem influência positiva no desempenho dos estudantes, como verificado nos resultados desta pesquisa.

No caso de etnia, os estudantes de etnia branca obtiveram melhores resultados, conforme o Teste Kruskal-Wallis aplicado aos dados, e que possibilitou demonstrar a existência de discrepância entre as diferentes etnias. Dessa análise, aceita-se a H3, a qual resultou em um valor de $\chi 2$ igual a 65,817, com um valor $p \leq 0,01$. Os resultados encontrados de que os estudantes de etnia branca obtiveram melhores resultados corroboram os achados da literatura (DEFREITAS; RINN, 2013; WALTON; COHEN, 2011). Entretanto, torna-se importante refletir sobre as origens das desigualdades que determinados grupos étnicos enfrentam na sociedade e que afetam seus desempenhos (WALTON; COHEN, 2011).

Os achados para horas de estudo demonstram que essa variável influenciou o desempenho, sendo que os estudantes que obtiveram maiores notas foram os que dedicaram mais de três horas de estudo semanais. Por meio do Teste Kruskal-Wallis, o valor de $\chi 2$ foi igual a 54,751, com um valor $\mathrm{p} \leq 0,01$, confirmando a H6.

Referente à variável horas de trabalho, constatou-se, por meio do Teste KruskalWallis, que a dimensão 'trabalhar vinte ou mais horas' apresenta maior influência no desempenho. Com base nesses resultados da Tabela 3, $\chi 2$ foi igual a 12,439, com um valor $\mathrm{p} \leq 0,01$, aceitando a H8.

No caso das horas de estudo e horas de trabalho, esses como fatores acadêmicos, notou-se que os estudantes que dedicavam mais de três horas de estudo semanais e que trabalhavam vinte horas ou mais apresentaram melhor desempenho. Estudos anteriores também que encontraram correlação entre os fatores acadêmicos e o desempenho dos estudantes (CALLENDER, 2008; DURÁN SANTOMIL et al., 2016; STATER, 2009).

A região de funcionamento dos cursos de administração pública na qual os estudantes apresentaram melhor desempenho foi a Sudeste, corroborando pesquisa anterior (CARMO; ALMEIDA, 2015). As dimensões desta variável apresentaram grande discrepância por meio do Teste Kruskal-Wallis, tendo um valor de $\chi 2$ igual a 497,093, com um valor $\mathrm{p} \leq 0,01$. Confirma-se, assim, a H10.

A variável gênero apresentou diferenças significativas a um nível de $1 \%$ nos resultados obtidos no Enade pelos estudantes do gênero masculino. Sendo estes os que obtiveram melhores notas por terem um intervalo médio superior ao das mulheres quando 
se aplica o Teste U de Mann-Whitney e o estatístico W da soma das escalas de Wilcoxon $(\mathrm{Z}=-10,667 ; \mathrm{p}$-valor $\leq 0,01)$. Assim, a H4 foi confirmada.

Em relação a idade para um nível de significância de 5\%, constatou-se que estudantes abaixo da média de idade (32,696 anos) tiveram um desempenho superior aos com idade acima da média. Esse resultado foi obtido por meio do Teste U de Mann-Whitney e o estatístico $\mathrm{W}$ da soma das escalas de Wilcoxon $(\mathrm{Z}=-2,425$; $\mathrm{p}$-valor $\leq 0,05)$. Dessa forma, aceita-se a H5.

Sobre os resultados encontrados para as variáveis de identificação, gênero e idade, os estudantes do gênero masculino do curso de administração pública obtiveram melhores resultados no Enade, e os estudantes abaixo da média de idade (32,696 anos) tiveram um desempenho superior aos com idade acima da média. A literatura indica que, dependendo das áreas e cursos, as notas mais elevadas podem ser dos homens ou das mulheres (SILVA; VENDRAMINI; LOPES, 2010). No que concerne à idade, pesquisas anteriores demonstraram que os estudantes mais jovens possuem melhor desempenho do que estudantes mais velhos (CARMO; ALMEIDA, 2015; MORICONI; NASCIMENTO, 2014).

Os alunos que tiveram financiamento apresentaram melhor desempenho no Enade 2015 do que os alunos sem financiamento, corroborando achados de outras pesquisas (CALLENDER, 2008; DURÁN SANTOMIL et al., 2016; STATER, 2009). Esta conclusão confirma a H7, com base no Teste U de Mann-Whitney e o estatístico W da soma das escalas de Wilcoxon $(Z=-1,812$; $p$-valor $\leq 0,1)$.

Os estudantes de cursos de administração pública em IES privadas obtiveram melhores notas no Enade do que os estudantes de IES públicas. Aplicou-se para a variável categoria administrativa o Teste U de Mann-Whitney e o estatístico W da soma das escalas de Wilcoxon $(Z=-4,102$; p-valor $\leq 0,01)$, aceitando-se a H9. A literatura indicou para a categoria administrativa apresentar relação com o desempenho dos estudantes no Enade (CREPALDE; SILVEIRA, 2016).

\section{Conclusões}

O objetivo do artigo foi analisar os fatores que influenciam o desempenho acadêmico dos alunos dos cursos de graduação em administração pública no Brasil. Para tanto, utilizou-se dados secundários do Enade 2015. Esta pesquisa de abordagem quantitativa baseou-se em técnicas estatísticas, como correlação bivariada e análise univariada.

Os fatores encontrados na literatura como influenciadores do desempenho acadêmico foram socioeconômicos (renda familiar, escolaridade dos pais, profıssão dos pais, etnia), de identificação (gênero, idade), acadêmicos (horas de estudo, financiamento, horas de trabalho) e de características das IES e cursos, como categoria administrativa e região do curso.

Em relação à análise bivariada, encontrou-se que a nota bruta da prova está correlacionada positivamente com a renda, escolaridade dos pais, gênero, horas de estudo e categoria administrativa, e correlacionada negativamente com etnia, idade, horas de trabalho e região de funcionamento do curso.

Posteriormente, a análise univariada confirmou as hipóteses de pesquisa, indicando relação entre o desempenho acadêmico e renda, escolaridade dos pais, etnia, gênero idade, 
horas de estudo, financiamento, horas de trabalho, categoria administrativa e região de funcionamento do curso.

Contatou-se que fatores socioeconômicos como renda e escolaridade dos pais influenciaram positivamente o desempenho acadêmico, ou seja, quanto maior a renda familiar e a escolaridade dos pais melhor foi o desempenho no Enade dos estudantes dos cursos de administração pública. Esses resultados sugerem o quanto os âmbitos sociais e econômicos podem impactar a trajetória acadêmica e também profissional, pois trata-se de estudantes concluintes, que já estão inseridos no mercado de trabalho ou estão em busca das primeiras oportunidades. Chama-se atenção para a realidade brasileira, na qual há grande desigualdade social, por conta da má distribuição de renda e não oportunidade de estudo para todos, pela necessidade de trabalho remunerado. Essa desigualdade transparece nos caminhos escolares e universitários, em que estudantes com boas condições econômicas tendem a frequentar as melhores escolas e instituições de ensino superior.

Outro resultado que traz reflexão refere-se aos estudantes de etnia branca que obtiveram melhores resultados no Enade 2015. Sobre a etnia, deve-se buscar compreender as origens das desigualdades que determinados grupos étnicos enfrentam na sociedade e que afetam seus desempenhos (WALTON; COHEN, 2011).

Quanto ao gênero dos estudantes, os homens obtiveram melhor desempenho do que as mulheres. Entretanto, a teoria indica que, dependendo das áreas e cursos, as notas mais elevadas podem ser de homens ou mulheres (SILVA; VENDRAMINI; LOPES, 2010). Observou-se ainda que um número mais elevado horas semanais dedicadas ao estudo, a existência de financiamento (bolsa de estudos) e a localização dos cursos de na região Sudeste influenciaram positivamente o desempenho. Dessa forma, notou-se que a dedicação dos estudantes que buscaram estudar semanalmente mais do que três horas contribuiu para um bom desempenho no Enade, que testa conhecimentos gerais e específicos da área do curso. Por outro lado, está a importância do financiamento para aqueles estudantes que não possuem condições financeiras de arcar com as despesas das instituições privadas.

Por fim, constatou-se que estudantes com idade abaixo da média e de cursos de IES privadas obtiveram melhores notas no Enade.

Este estudo busca contribuir do ponto de vista teórico para o entendimento de quais fatores podem ter influenciado o desempenho de estudantes dos cursos de administração pública no Enade 2015, além de fornecer informações que podem auxiliar as instituições de ensino superior a buscar estratégias que visem melhorar o desempenho de seus estudantes.

\section{Referências}

ALBERNAZ, Ângela; FERREIRA, Francisco; FRANCO, Creso. Qualidade e eqüidade no ensino fundamental brasileiro. Pesquisa e Planejamento Econômico, Rio de Janeiro, v. 32, n. 3, p. 453-476, 2002.

ALMEIDA, Leandro et al. Sucesso e insucesso no ensino básico: relevância de variáveis sócio-familiares e escolares em alunos do 50 ano. In: CONGRESSO GALAICO PORTUGUÊS DE PSICOPEDAGOGIA, 8., 2005, Braga. Actas... Braga: Universidade do Minho, 2005. p. 3629-3642. 
ALMEIDA, Leandro et al. Acesso e sucesso no ensino superior em Portugal: questões de género, origem sócio-cultural e percurso académico dos alunos. Psicologia, Porto Alegre, v. 19, n. 3, p. 507-514, 2006.

ANDRIETTI, Vicenzo; VELASCO, Carlos. Lecture attendance, study time, and academic performance: a panel data study. The Journal of Economic Education, London, v. 46, n. 3, p. 239-259, 2015.

BAE, Dayoung; WICKRAMA, Kandauda A. S. Family socioeconomic status and academic achievement among Korean adolescents: linking mechanisms of family processes and adolescents' time use. Journal of Early Adolescence, v. 35, n. 7, p. 1014-1038, 2015.

BARBOSA, Maria Eugénia Ferrão; FERNANDES, Cristiano. A escola brasileira faz diferença? Uma investigação dos efeitos da escola na proficiência em matemática dos alunos da 4a série. In: FRANCO, Creso (Ed.). Promoção, ciclos e avaliação na educação. Curitiba: ArtMed, 2001. p. 1-23.

BENAVENTE, Ana. Insucesso escolar no contexto português: abordagens, concepções e políticas. Análise Social, Lisboa, v. 25, n. 108/109, p. 715-733, 1990.

BERNSTEIN, Basil. Class, codes and control: Towards a theory od educational transmission. v. 3. London: Routledge; New York: Taylor \& Francis, 2003.

BERTOLIN, Júlio C. G.; MARCON, Telmo 0 (des)entendimento de qualidade na educação superior brasileira: das quimeras do provão e do Enade à realidade do capital cultural dos estudantes. Avaliação, Campinas, v. 20, n. 1, p. 105-122, 2015.

BRASIL. Instituto Nacional de Estudos e Pesquisas Educacionais Anísio Teixeira. Exame Nacional de Desempenho dos Estudantes - ENADE. Brasília, DF: Inep, 2015. Disponível em: <http://portal.inep.gov.br/ enade>. Acesso em: 19 set. 2016.

BRASIL. Ministério da Educação. Saiba como funciona sistema de ensino superior no Brasil. Brasília, DF: MEC, 2009.

BRITES-FERREIRA, José et al. (In)sucesso académico no ensino superior: conceitos, factores e estratégias de intervenção. Revista Iberoamericana de Educación Superior, Huixquilucan, v. 2, n. 4, p. 28-40, 2011.

BUCHMANN, Claudia; DIPRETE, Thomas A. The growing female advantage in college completion: the role of family background and academic achievement. American Sociological Review, v. 71, p. 515-541, 2006.

CALLENDER, Claudia. The impact of term: time employment on higher education students' academic attainment and achievement. Journal of Education Policy, London, v. 23, n. 4, p. 359-377, 2008.

CARM0, Carlos Roberto Souza; ALMEIDA, Sirlene de Aguiar Fernandes. Enade: a influência de variáveis qualitativas no desempenho dos alunos dos cursos de ciências contábeis do Brasil. Revista de Auditoria, Governança e Contabilidade, Monte Carmelo, v. 3, n. 7, p. 71-87, 2015.

CARNEY, Claire; McNEISH, Sharon; McCOLL, John. The impact of part time employment on students' health and academic performance: a Scottish perspective. Journal of Further and Higher Education, London, v. 29, n. 4, p. 307-319, 2005. 
Jaqueline Zermiani BRANDT; Francisca TEJEDO-ROMERO; Joaquim Filipe Ferraz Esteves ARAUJO

CÉSAR, Cibele Comini; SOARES, José Francisco. Desigualdades acadêmicas induzidas pelo contexto escolar. Revista Brasileira de Estudos de População, Belo Horizonte,v. 18, n. 1/2, p. 97-110, 2001.

COLEMAN, James et al. Equality of educational opportunity U.S. Department of Health, Education and Welfare. [S. I.: s. n.], 1966.

COONROD, Lane. The effects of financial aid amounts on academic performance. The Park Place Economist, Bloomington, v. 16, p. 24-35, 2008.

CORIA, Elizabeth; HOFFMAN, John L. Financial aid tipping points: an analysis of aid and academic achievement at a California community college. Community College Journal of Research and Practice, London, v. 40, n. 2, p. 160-170, 2016.

CREPALDE, Neylson João Batista Filho; SILVEIRA, Leonardo Souza. Desempenho universitário no Brasil: estudo sobre desigualdade educacional com dados do Enade 2014. Revista Brasileira de Sociologia, Aracajú, v. 4, n. 7, p. 211-238, 2016.

DAROLIA, Rajeev. Working (and studying ) day and night: Heterogeneous effects of working on the academic performance of full-time and part-time students. Economics of Education Review, Amsterdã, v. 38, p. 38-50, 2014.

DEFREITAS, Stacie Craft; RINN, Anne. Academic achievement in first generation college students : the role of academic self-concept. Journal of the Scholarship of Teaching and Learning, Bloomington, v. 13, n. 1, p. 57-67, 2013.

DURÁN SANTOMIL, Pablo et al. Determinantes del rendimiento académico del alumnado de una asignatura de contabilidad : el caso de la USC. Revista de Docencia Universitaria, Valência, v. 14, n. 1, p. 151-178, 2016.

FAGUNDES, Caterine Vila. Percepção dos estudantes universitários acerca do acesso à educação superior: um estudo exploratório. Revista Brasileira de Estudos Pedagógicos, Brasília, DF, v. 95, n. 241, p. 508-525, 2014.

FERNANDES, António Sousa. 0 insucesso escolar. In: PIRES, Eurico Lemos; FERNANDES, António Sousa; FORMOSINHO, João (Ed.). A construção social da educação escolar. Porto: ASA, 1991. p. 187-213.

GUJARATI, Damodar N. Basic econometrics. 3. ed. New York: McGraw-Hill, 1995.

LEMOS, Karinne Custódio Silva; MIRANDA, Gilberto José. Alto e baixo desempenho no enade: que variáveis explicam? Revista Ambiente Contábil, Natal, v. 7, n. 2, p. 101-118, 2015.

LEMOS, Valter. Políticas públicas de educação: equidade e sucesso escolar. Sociologia, Problemas e Práticas, Lisboa, v. 73, p. 151-169, 2013.

MILLS, Christina et al. Factors associated with the academic success of first year health science students. Advances in Health Sciences Education, v. 14, n. 2, p. 205-217, 2009.

MORIANA, Juan Antonio et al. Extracurricular activities and academic performance in secondary students. Electronic Journal of Research in Educational Psychology, Almera, v. 4, n. 8, p. 35-46, 2006. 
MORICONI, Gabriela Miranda; NASCIMENTO, Paulo Augusto Meyer Mattos. Fatores associados ao desempenho dos concluintes de engenharia no Enade 2011. Estudos em Avaliação Educacional, São Paulo, v. 25, n. 57, p. 248-278, 2014.

MÜLLER, Helne; PRINSL00, Paul; DU PLESSIS, Anneki. Validating the profile of a successful first year accounting student. Meditari Accountancy Research, Bingley, v. 15, n. 1, p. 19-33, 2007.

PALERMO, Gabrielle A.; SILVA, Denize Britz do Nascimento; NOVELLINO, Maria Salet Ferreira. Fatores associados ao desempenho escolar: uma análise da proficiência em matemática dos alunos do 50 ano do ensino fundamental da rede municipal do Rio de Janeiro. Revista Brasileira de Estudos de População, Rio de Janeiro, v. 31, n. 2, p. 367-394, 2014.

PÉREZ-CÁRCELES, Maria Concepción et al. El género como variable moderadora de los resultados académicos en la enseñanza universitaria. Regional and Sectoral Economic Studies, v. 14, n. 3, p. 55-64, 2014.

PINTO, António Bernardo Moraes; DELGAD0, João Paulo Ferreira; MARTINS, Alcina Manoela de Oliveira. Significados e perspectivas do insucesso escolar no ensino profissional em Portugal. Revista Cadernos de Educação, Pelotas, n. 51, p. 1-21, 2015.

POKROPEK, Artur; BORGONOVI, Francesca; JAKUBOWSKI, Maciej. Socio-economic disparities in academic achievement: a comparative analysis of mechanisms and pathways. Learning and Individual Differences, Amsterdã, v. 42, p. 10-18, 2015.

SAAVEDRA, Luísa. Sucesso/Insucesso escolar: a importância do nível socioeconômico e do gênero. Psicologia, Fortaleza, v. 15, n. 1, p. 67-92, 2001.

SCOTT-CLAYTON, Judith. The causal effect of federal work-study participation: quasi-experimental evidence from West Virginia. Educational Evaluation and Policy Analysis, New Bury Park, v. 33, n. 4, p. 506-527, 2011.

SILVA, Marjorie Cristina Rocha da; VENDRAMINI, Claudette Maria Medeiros; LOPES, Fernanda Luzia. Diferenças entre gênero e perfil sócio-econômico no Exame Nacional de Desempenho do Estudante. Avaliação, Campinas, v. 15, n. 3, p. 185-202, 2010.

SIRIN, Selcuk R. Socioeconomic status and academic achievement: a meta-analytic review of research. Review of Educational Reserach, State College, v. 75, p. 417-453, 2005.

STATER, Mark. The impact of financial aid on college GPA at three flagship public institutions. American Educational Research Journal, v. 46, n. 3, p. 782-815, 2009.

STEENKAMP, Lee P.; BAARD, Roelof S.; FRICK, Liezel B. Factors influencing success in first-year accounting at a South African university : a comparison between lecturers' assumptions and students' perceptions. SA Journal of Accounting Research, v. 23, n. 1, p. 113-140, 2009.

SULEYMANOV, Farid. Relationship between teacher assistant support and academic achievements of exceptional students in inclusive education. The Online Journal of New Horizons in Education, v. 6, n. 2, p. 93-100, 2016. 
TEJEDOR, Francisco Javier. Poder explicativo de algunos determinantes del rendimiento en los estudios universitarios. Revista Española de Pedagogía, Madrid, v. 61, n. 224, p. 5-32, 2003.

TEJEDOR, Francisco Javier; MUÑOZ-REPISO, Ana García-Valcárcel. Causas del bajo rendimiento del estudiante universitario (en opinión de los profesores y alumnos): propuestas de mejora en el marco del EEES. Revista de Educación, Madrid, n. 343, p. 443-473, 2007.

TOMÉ, Francisco José Sanches. Factores socioeconómicos e sucesso educativo. Revista Interdisciplinar de Ciências Sociais e Humanas, Matosinhos, v. 1, n. 1992, p. 26-38, 2013.

WALTON, Gregory M.; COHEN, Geoffrey L. A brief social-belonging intervention improves academic and health outcomes of minority students. Science, Washington, DC, v. 331, p. 1447-1451, 2011.

WHITE, Karl R. The relation between socioeconomic status and academic achievement. Psychological Bulletin, Washington, DC, v. 91, n. 3, p. 461-481, 1982.

Recebido em: 06.06.2018

Revisado em: 26.02.2019

Aprovado em: 19.03.2019

Jaqueline Zermiani Brandt é mestre em administração pública pela Universidade do Minho, Portugal e graduada em administração pela Universidade Federal de Santa Catarina.

Francisca Tejedo-Romero é PhD em contabilidade pela Universidade de Castilla - La Mancha, Espanha. Professora contratada doutora na Universidade de Castilla - La Mancha.

Joaquim Filipe Ferraz Esteves Araujo é PhD em Administração Pública, pela University of Exeter, Reino Unido. É Professor Catedrático na Universidade do Minho, Portugal, e no Centro de Investigação em Ciência Política-CICP (UID/CPO/0758/2019). 